\title{
Congenital Hypothyroidism: A Severely Neglected Case Report in Bangladesh
}

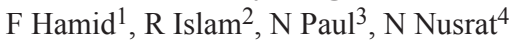

\begin{abstract}
:
A 6 year old girl presented with not growing well, delayed milestone of development, feeding difficulty, constipation since birth and breathing difficulty for last 3 months. The diagnosis was congenital hypothyroidism which was confirmed by Serum FT4 \& TSH. The patient was put on oral thyroxin and showed little improvement initially and discharged on request after 15 days but unfortunately she died after 1 month at her residence.
\end{abstract}

\section{Introduction :}

Congenital hypothyroidism is inadequate thyroid hormone production in newborn infants. This can occur because of an anatomic defect in the gland, an inborn error of thyroid metabolism or iodine deficiency. ${ }^{1}$ The incidence of congenital hypothyroidism, as detected through newborn screening, is approximately 1 per 4000 births. ${ }^{2,3}$ Thyroid hormone is necessary for normal brain growth and myelination and for normal neuronal connections. The most critical period for the effect of thyroid hormone on brain development is the first few months of life. ${ }^{1}$ Profound mental retardation is the most serious effect of untreated congenital hypothyroidism. Severe impairment of linear growth and bone maturation also occurs. ${ }^{6}$ Diagnosis of congenital hypothyroidism is confirmed by demonstrating decreased levels of serum thyroid hormone (total or free T4) and elevated levels of thyroid-stimulating hormone (TSH). ${ }^{9}$ Thyroid scanning (using technetium-99m or iodine-123) may be useful in defining the cause of hypothyroidism and may aid in genetic counseling. ${ }^{11}$ Thyroid scans can also demonstrate the presence of an ectopic thyroid, such as a lingual or sublingual gland. Ultrasonography may be a reasonable alternative or addition to scintigraphy but may fail to reveal some ectopic glands. ${ }^{11}$ The mainstay in the treatment of congenital hypothyroidism is early diagnosis and thyroid hormone replacement. ${ }^{12}$

1. Dr. Farzana Hamid, Assistant Professor, Dept. of Paediatrics, Delta Medical College, Dhaka.

2. Dr. Rafiqul Islam, Professor, Dept. of Paediatrics, Delta Medical College, Dhaka.

3. Dr. Nibedita Paul, Resident Physician, Dept. of Paediatrics, Delta Medical College, Dhaka.

4. Dr. Nadia Nusrat, Assistant Registrar, Dept. of Paediatrics, Delta Medical College, Dhaka.

\section{Corresponding Author:}

Dr. Farzana Hamid, Assistant Professor, Dept. of

Paediatrics, Delta Medical College, Dhaka

\section{Case Report:}

The patient was a 6 years old girl from Pirozpur, Bangladesh got admitted in Delta Medical College and Hospital with the complaints of not growing well since birth, delayed milestone of development, feeding difficulty, constipation

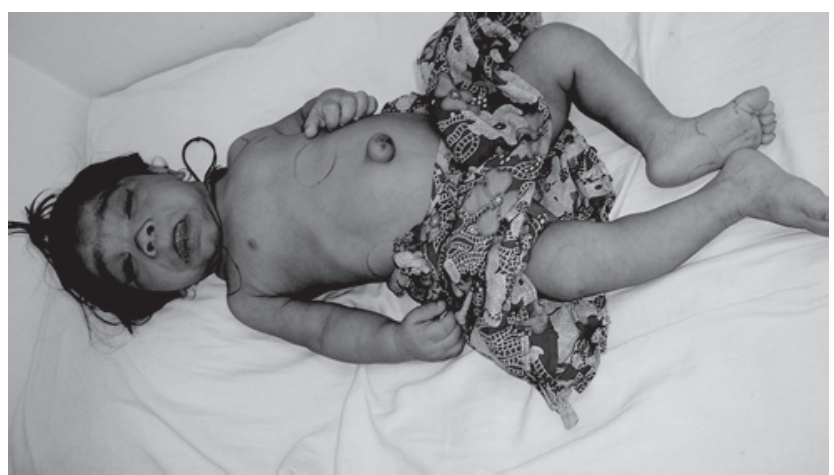

Fig. 1: Showed features of congenital hypothyroidism

since birth and respiratory distress for last 3 months. She was delivered full term by vaginal delivery. Her antenatal and postnatal period was uneventful with a birth weight of 2.7 $\mathrm{kg}$. She was exclusively breast fed for 6 months. Then complementary feeding was given and now on semisolid food. Mother had no illness. She had achieved social smile at 2.5 months and partial neck control at 1 year of age. She could not say any word. On examination, she was breathless at rest, had coarse facial features with wrinkling of forehead, narrow palpebral fissure, absent teeth, depressed nasal bridge and a protruding tongue. She had wide open anterior fontanel $(4 \times 4 \mathrm{~cm})$ with dry and thick skin. Abdomen was distended with umbilical hernia. She had short, broad hand with short fingers. Her anthropometry was still of infantile proportion with a length of $71 \mathrm{~cm}(\mathrm{WHZ}=-9)$, weight of $8.7 \mathrm{~kg}(\mathrm{WAZ}=$ -5 ), upper and lower segment ratio $1.4: 1$. There was no organomegaly or no goiter. She had hypotonia with delayed relaxation of deep tendon reflex. Heart sounds were distant. She had partial neck control only. She had only some babbling of sound. Vision and hearing was intact. Other systemic examination was normal.

Laboratory investigations showed hemoglobin $-6.4 \mathrm{gm} / \mathrm{dl}$, ESR-36 mm in 1st hour, WBC-5500/cu mm with $43 \%$ polymorph, $51 \%$ lymphocytes. PBF showed anisopoikilocytosis with eliptocytes. Her thyroid profile was suggestive of hypothyroidism: FT4- $<0.64 \mathrm{pmol} / \mathrm{L}$ [Normal 10.32 - 25.8], S TSH $>75 \mathrm{miu} / \mathrm{ml}$ [Normal 0.4 - 4]. USG of thyroid was normal. Chest X-ray showed enlarged cardiac shadow and epiphyseal dysgenesis of humerus. A 2D echocardiography revealed moderate pericardial effusion and ECG showed low voltage tracing with sinus bradicardia. Radionuclide scan showed normal uptake. 


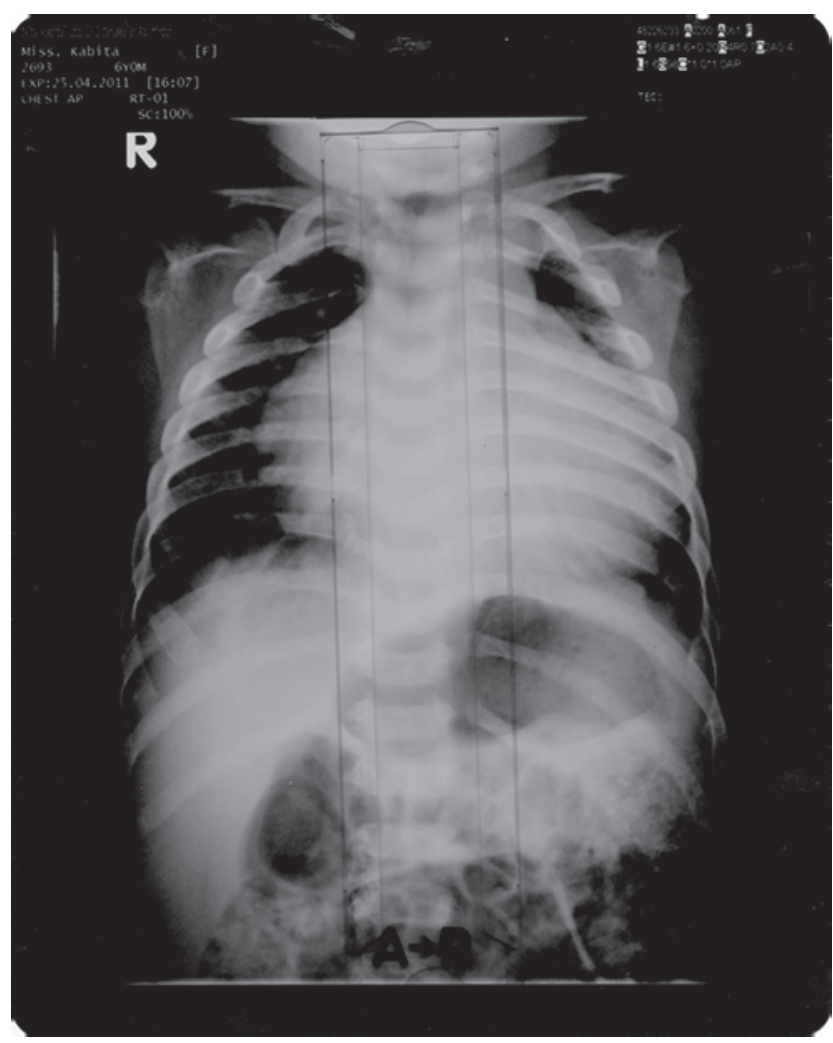

Fig. 2: Chest X-ray showed cardiomegaly and epiphyseal dysgenesis of humerus.

Thus she was diagnosed as congenital hypothyroidism with pericardial effusion, most likely due to dyshormonogenesis. Thyroxin was started at a dose of $4 \mu \mathrm{g} / \mathrm{kg} /$ day with other supportive treatment and her respiratory distress improved and heart size decreased after 15 days and she was discharged on request. After 1 month she died at her residence.

\section{Discussion :}

The thyroid gland is a butterfly-shaped tissue in the lower neck. It makes iodine-containing hormones that play an important role in regulating growth, brain development, and body metabolism. Congenital hypothyroidism occurs when the thyroid gland fails to develop or function properly. ${ }^{1}$ In 80 to 85 percent of cases, the thyroid gland is absent, abnormally located, or hypoplastic. In the remaining cases, a normal-sized or enlarged thyroid gland is present, but production of thyroid hormones is decreased or absent (dyshormonogenesis). ${ }^{2}$ Family history should be carefully reviewed for information about similarly affected infants or family members with unexplained mental retardation. ${ }^{3}$

Maternal history of a thyroid disorder and mode of treatment, whether before or during pregnancy, can occasionally provide the etiology of the infant's problem. ${ }^{3,4}$ Radioactive iodine therapy of pregnant women may cause permanent congenital hypothyroidism. Congenital hypothyroidism is more common in infants with birth weight less than 2,000 g or more than 4,500 g. Congenital hypothyroidism is more common in multiple births. ${ }^{5}$

Infants with congenital hypothyroidism are usually identified within the first 2-3 weeks of life. ${ }^{1}$ Congenital hypothyroid child may present with slightly increased head size due to myxedema of the brain. There may be prolongation of physiological jaundice, lethargy, somnolence, large tongue and nasal obstruction. Affected infants cry little, sleep more and are very lethargic. There may be presence of umbilical hernia, hypothermia, constipation, edema of genitals and extremities, cardiomegaly, bradycardia and asymptomatic pericardial effusion. ${ }^{6}$ As the child grows, infantile proportions are maintained and child may have disproportionate short stature. Both anterior and posterior fontanelles are wide-open and coarse facies such as hypertelorism, depressed nasal bridge, puffiness of eyes, open mouth and short neck develop. Skin may become dry, rough. Hairline reaches far down on forehead and development is retarded. Voice is hoarse and child has hypotonia.1,6 Goiter may be seen in patients with dyshormonogenesis, thyroid hormone resistance and transient hypothyroidism. A small but significant number (3-7\%) of infants with congenital hypothyroidism have other birth defects, mainly atrial and ventricular septal defects. ${ }^{7}$

The morbidity from congenital hypothyroidism can be reduced to a minimum by early diagnosis and treatment. ${ }^{8}$ Although initial preliminary studies were performed using thyroid-stimulating hormone (TSH) levels in cord blood, mass screening was made feasible by the development of radioimmunoassay for TSH and thyroxine (T4) from blood spots on filter paper, obtained for neonatal screening tests. ${ }^{9}$ Diagnosis of congenital hypothyroidism is confirmed by demonstrating decreased levels of serum thyroid hormone (total or free T4) and elevated levels of thyroid-stimulating hormone (TSH). If maternal antibody-mediated hypothyroidism is suspected, maternal and neonatal antithyroid antibodies may confirm the diagnosis. ${ }^{10}$ Such antibodies are an uncommon cause of congenital hypothyroidism. Low or low-normal serum total T4 levels in the setting of a serum TSH within the reference range suggests TBG deficiency. This congenital disorder causes no pathologic consequence; however, it should be recognized to avoid unnecessary thyroid hormone administration. ${ }^{10}$

Thyroid scanning (using technetium-99m or iodine-123) may be useful in defining the cause of hypothyroidism and may aid in genetic counseling. ${ }^{11}$ The absence of radionuclide uptake suggests sporadic athyreotic hypothyroidism but can also be seen when uptake is blocked by excess iodide or thyroid receptor blocking antibodies. If no uptake is found on isotope scanning, thyroid ultrasonography may demonstrate thyroid tissue in these patients. One study of 210 scanned infants stated a preference for using iodine-123 over pertechnetate. ${ }^{11}$ Thyroid scans can also demonstrate the presence of an ectopic thyroid, such as a lingual or sublingual gland, which is also sporadic. Ultrasonography may be a reasonable alternative or addition to scintigraphy but may fail to reveal some ectopic glands. ${ }^{11}$ A lateral radiograph of the knee may be obtained to look for the distal femoral epiphysis. This ossification center appears at about 36 weeks' gestation. Its absence in a term or post term infant indicates prenatal effects of hypothyroidism. ${ }^{10}$ 
The mainstay in the treatment of congenital hypothyroidism is early diagnosis and thyroid hormone replacement for life. One study suggested that optimal care includes diagnosis before age 13 days and normalization of thyroid hormone blood levels by age 3 weeks. ${ }^{12}$ Appropriate psychological, developmental, and educational evaluations should also be considered. Dietary iodide supplementation in iodine-deficient areas can prevent endemic cretinism but does not have a major effect on sporadic congenital hypothyroidism. ${ }^{13}$ Children with congenital hypothyroidism should be monitored clinically and biochemically. Laboratory measurements of T4 (total or free T4) and TSH should be repeated 4-6 weeks after initiation of therapy, then every 1-3 months during the first year of life and every 2-4 months during the second and third years. In children aged 3 years and older, the time interval between measurements may be increased. ${ }^{14}$ Formal developmental and psycho neurological evaluations should be considered in all infants with congenital hypothyroidism. Such evaluations are especially important in children whose treatment was delayed or inadequate. ${ }^{15,16}$

Our patient, a very unfortunate girl presented lately with all features of severe congenital hypothyroidism with moderate pericardial effusion. We could not determine the cause of death as the patient died one month after discharging from hospital.

\section{Conclusion :}

Early diagnosis and treatment of congenital hypothyroidism

1. Kurinczuk JJ, Bower C, Lewis B, Byrne G. Congenital hypothyroidism in Western Australia 1981-1998. J

Paediatr Child Health. Apr 2002;38(2):187-91.

2. Klett M. Epidemiology of congenital hypothyroidism. Exp Clin Endocrinol Diabetes. 1997;105 Suppl

3. 4:19-23.

Jalil MQ, Mia MJ, Ali SM. Epidemiological study of endemic cretinism in a hyperendemic area.

4. Bangladesh Med Res Counc Bull. Apr 1997;23(1):34-7.

Medda E, Olivieri A, Stazi MA, et al. Risk factors for congenital hypothyroidism: results of a population

5. casecontrol study (1997-2003). Eur J

Endocrinol. Dec

2005;153(6):765-73.

Olivier i A, Medda E, De Angelis S, Valensise H, De Felice M, Fazzini C, et al. High risk of congenital hypothyroidism in multiple pregnancies. J Clin Endocrinol Metab. Aug 2007;92(8):3141-7.
6. Lowery GH, Aster RH, Carr EA, Ramon G, Beierwates WH, Spafford NR. Early Diagnostic Criteria of Congenital Hypothyroidism: A Comprehensive Study of Forty-Nine Cretins. Am J Dis Child. 1958;96(2):131-143.

7. Stoll C, Dott B, Alembik Y, Koehl C. Congenital

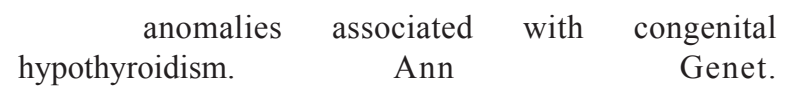

8. 1999;42(1):17-20.

Delange F. Screening for congenital hypothyroidism used as an indicator of the degree of iodine

9. deficiency and of its control. Thyroid. Dec 1998;8(12):1185-92.

Hertzberg V, Mei J, Therrell BL. Effect of laboratory

10. practices on the incidence rate of congenital 2:S48-53. hypothyroidism. Pediatrics. May 2010;125 Suppl

11. Eugster EA, LeMay D, Zerin JM, Pescovitz OH. Definitive diagnosis in children with congenital hypothyroidism. J Pediatr. May 2004;144(5):643-7.

Perry RJ, Maroo S, Maclennan AC, Jones JH, Donaldson

12. MD. Combined ultrasound and isotope scanning is more informative in the diagnosis of congenital

13. hypothyroidism than single scanning. Arch Dis Child. Dec 2006;91(12):972-6.

LaFranchi SH, Austin J. How should we be treating children with congenital hypothyroidism?. J

Pediatr Endocrinol Metab. May

14. 2007;20(5):559-78.

Jones JH, Donaldson MD. Audit of initial management of congenital hypothyroidism in the United Kingdom-comparison of UK

15. practice with European and UK guidelines. J Pediatr Endocrinol Metab. Nov 2009;22(11):1017-25.

Bongers-Schokking JJ, Koot HM, Wiersma D, et al.

16. Influence of timing and dose of thyroid development in infants with congenital hypothyroidism. J Pediatr. Mar 2000;136(3):292-7.

Selva KA, Harper A, Downs A, Blasco PA, Lafranchi $\mathrm{SH}$. Neurodevelopmental outcomes in congenital hypothyroidism: comparison of initial T4 dose and time to reach target T4 and TSH. J Pediatr. Dec 